\title{
TRANSITORY AMNESIA IS NOT ALWAYS PSYCHOGENIC
}

\author{
Isaac J. ${ }^{1}$, Viegas F. ${ }^{2}$, Gonçalves A. ${ }^{3}$, Vitor J. ${ }^{3}$, Marques J. ${ }^{3}$, Painho T. ${ }^{3}$, Simões R. ${ }^{3}$, Vale J. ${ }^{3}$ \\ 1 -Serviço de Psiquiatria Hospital José Joaquim Fernandes, Unidade Local de Saúde do Baixo Alentejo; 2 - Serviço de Psiquiatria \\ Hospital Fernando da Fonseca; 3 - Serviço de Neurologia Hospital Beatriz Ângelo
}

\section{Objectives}

The authors aim is to review the main differences between transient global amnesia (TGA) and psychiatric dissociative-like manifestations, based on a clinical case.

\section{Background}

Amnesia can occur transiently in neurological conditions such as TGA or transient epileptic amnesia, or may have a psychogenic basis. TGA is characterized by a sudden onset of acute anterograde amnesia with neither cognitive impairment nor altered consciousness, resolving within 24 hours without neurological physical signs.

\section{Materials and methods}

Clinical case description and non-systematic review of the literature.

\section{Clinical case description}

A 61-year-old woman, was admitted in the emergency room by a sudden episode in the workplace of memory loss described as malaise, repetitive questions and anterograde amnesia that lasted for six hours. At examination she was profoundly amnestic, with a retention span of three minutes and retrograde amnesia extending 20-30years in the past. Traumatic events/recent stress were denied. There was progressive improvement with total recovery in less than 24hours, only maintaining amnesic gap for events that occurred during those six hours. CT-scan, analysis, EKG, EEG, transthoracic echocardiogram and carotid ultrasound were normal. Brain MRI showed focal diffusion weighted signal changes in the hippocampus bilaterally.
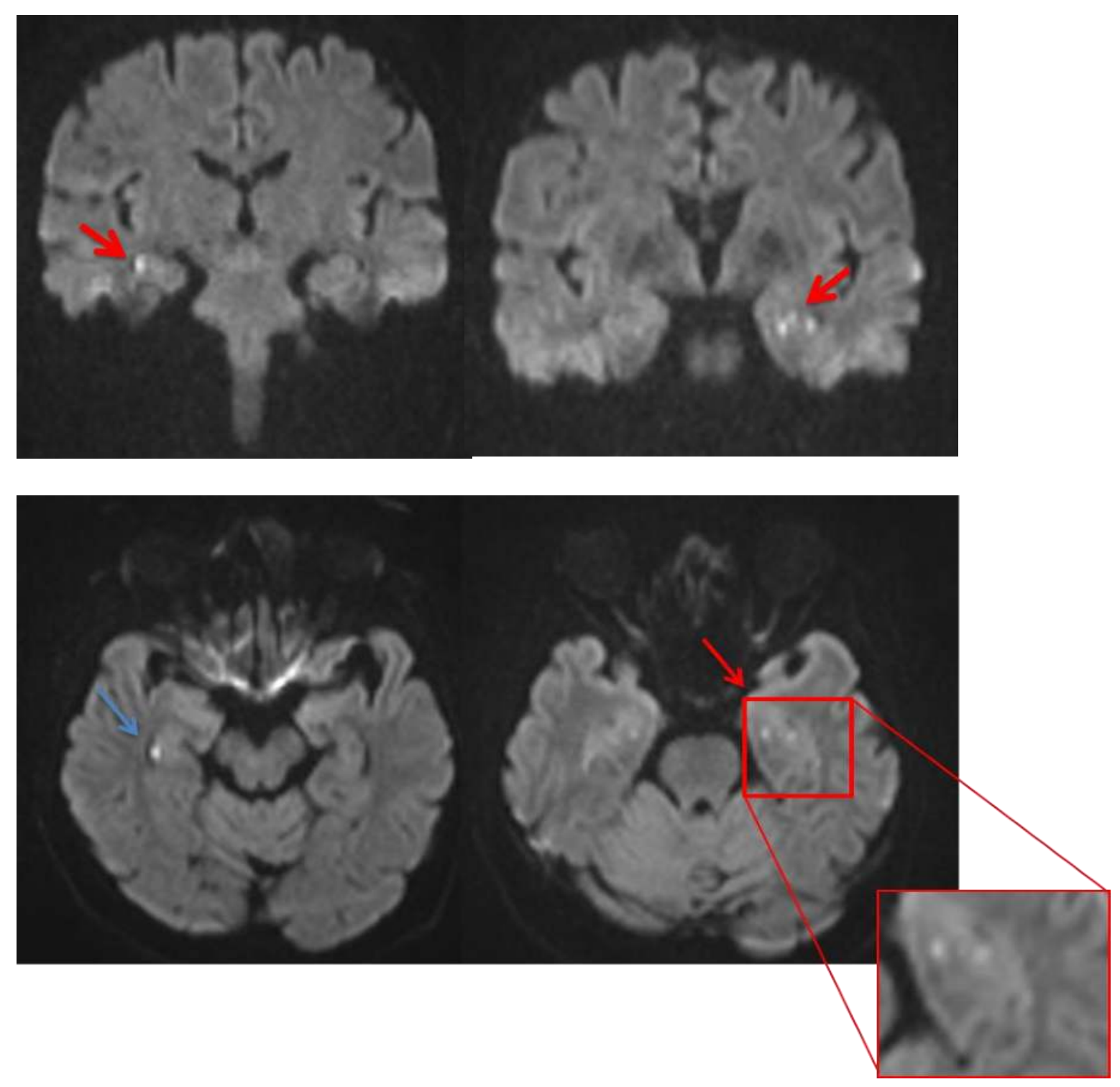

\section{Non-systematic review of the literature}

TGA is a clinical diagnosis. It was first described in 1956 as an "isolated episode of confusion with amnesia" not otherwise associated with other neurological deficits.

\section{Diagnostic Criteria for Transient Global Amnesia}

1. Attack must be witnessed and information available from a capable observer who was present for most of the attack

2. Clear-cut anterograde amnesia during the attack

3. Cognitive impairment limited to amnesia, without clouding of consciousness or loss of personal identity

4. No accompanying focal neurologic symptoms during the attack and no significant neurologic signs afterward

5. Absence of epileptic features

6. Resolution of the attack within $24 \mathrm{~h}$

Psychiatric comorbidity

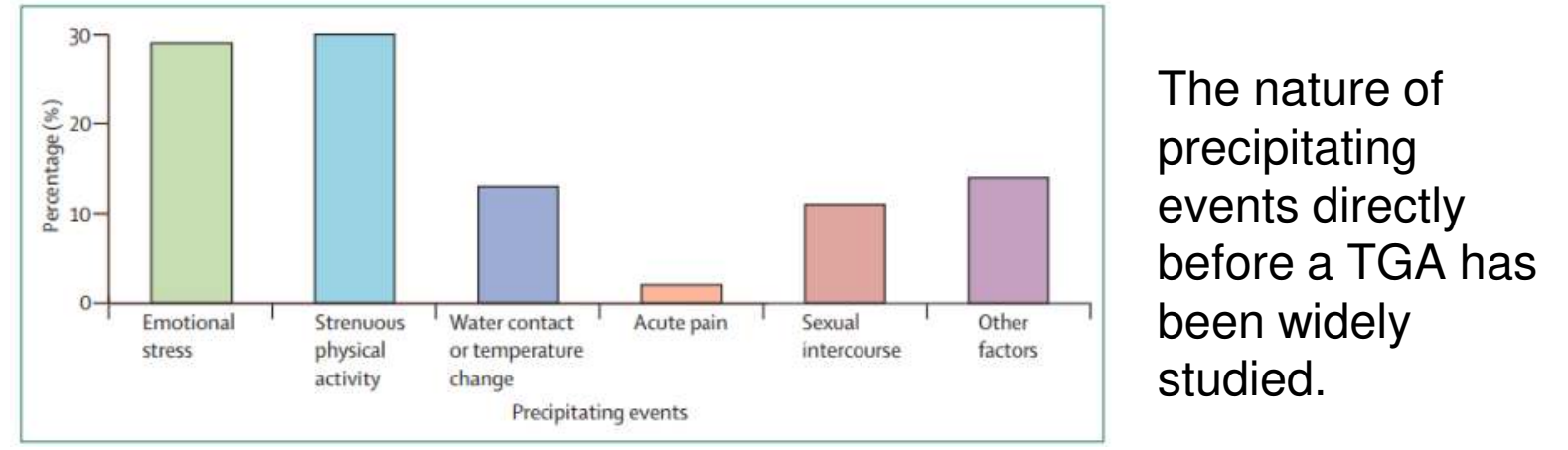

\section{Data from} epidemiological studies suggest that certain personality traits might be relevant in the aetiology of TGA.

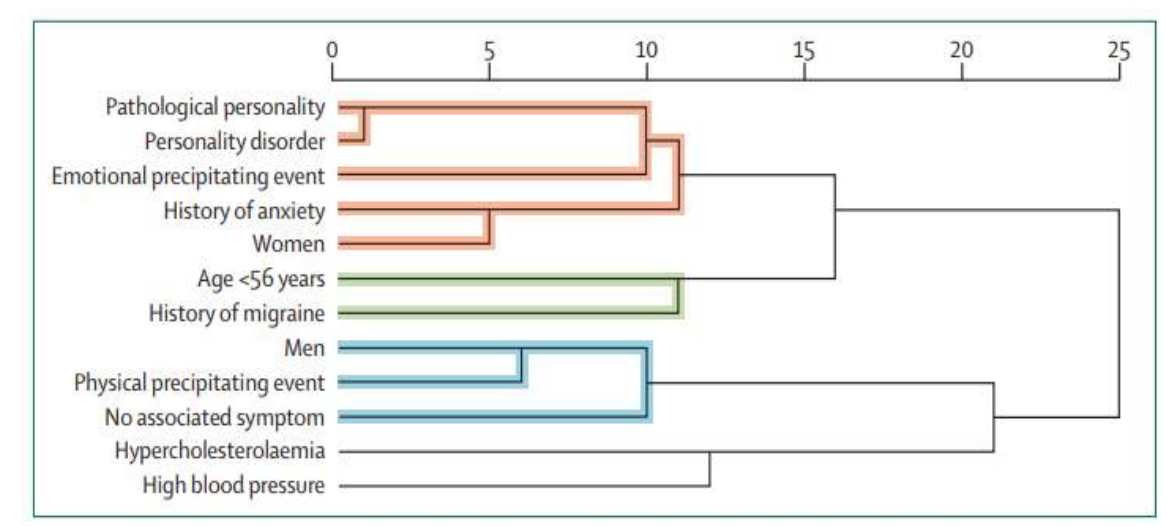

There is a limited, but important, range of differential diagnoses that might clinically mimic a TGA:

- Ischaemia in the posterior cerebral circulation

- Intoxication, adverse drug side-effects

- Complex focal seizures, transient epileptic amnesia, post-ictal conditions

- Post-traumatic amnesia

- Hypoglycaemia

\section{- Psychogenic amnesia, dissociative disorders}

Psychogenic amnesia, often labeled as dissociative amnesia, is differentiated from TGA, in which the former affects retrograde memory, it is generally episodic, and it is often accompanied by intense psychological distress.

\section{Conclusions}

TGA's etiology remains elusive and psychogenic amnesia may also be confused with this entity. The hippocampus, in TGA, has typically been sited as the affected structure. Also, in TGA, contrary to psychogenic amnesia, autobiographic data are recalled by patients and loss of memory is limited to one domain. 